\title{
Computational modelling and experimental validation of industrial forming processes by cold pressing of aluminum silicate powder
}

\author{
M. Penasa ${ }^{1}$, L. Argani ${ }^{2}$, D. Misseroni ${ }^{1}$, F. Dal Corso $^{1}$, \\ M. COVA ${ }^{3}$, AND A. PicCOLROAZ*1 \\ ${ }^{1}$ Dipartimento di Ingegneria Meccanica e Strutturale, Università di Trento, Italy \\ ${ }^{2}$ Department of Mathematical Sciences, University of Liverpool, UK \\ ${ }^{3}$ Ceramics $\&$ Tiles Technical Office, Sacmi Imola S.C., Italy
}

\begin{abstract}
A novel approach to the modelling and simulation of the industrial compaction process of ceramic powders is proposed, based on a combination of: (i) continuum mechanics modelling of the constitutive response of the material; (ii) finite element discretization and computer implementation of the mechanical model; (iii) parametric identification by a multi-objective optimization of simulated experimental tests. The capabilities of the proposed approach are highlighted through computer simulations of realistic industrial compaction processes, namely, the forming of an axisymmetric tablet and of a three-dimensional ceramic tile.

The presented methods and the pointed out results pave the way for the introduction of so-called virtual prototyping into the industrial practice of ceramic forming processes.
\end{abstract}

Keywords: Powder compaction; Parameter identification; Multi-objective optimization; Virtual prototyping

\section{Introduction}

Ceramic forming by cold pressing of powders is a common practice in both traditional and advanced ceramic technologies. Due to its industrial interest, this process has been the focus of much attention by the research community over the past decades. Two main approaches in the modelling of granular matter can be distinguished: a micromechanical approach, to analyse the deformation of individual granules in detail, and a continuum macroscopic approach, to describe averaged deformations at the macroscale.

The micromechanical approach has led to the development of the discrete element method (closely related to molecular dynamics) [1-5]. This method can accurately describe the granular flow during the first stage of the compaction process (low pressure), but it is excessively detailed

\footnotetext{
*Corresponding author: e-mail: roaz@ing.unitn.it; phone: +390461 282583.
} 
for later stages (high pressure) where the material is better described as a porous solid [6, 7]. Moreover, this method has the disadvantage that the size of the sample (number of particles) and the duration of a simulation are limited by the available computational power $[8,9]$. For this reason, the continuum macroscopic approach is preferable for the simulation of forming of large components. Moreover, the continuum material model can be implemented in a finite element computer code, which is more accessible to the industry than the discrete element method.

Based on the macroscopic approach, a new rational strategy is proposed in the present article for the computer simulation of real, large-scale ceramic component forming. The aim is to provide the ceramic manufacturers with an effective tool for the optimal design of moulds, punches, and presses for ceramic forming and, thus, to make virtual prototyping a realistic technology for the ceramic industry.

The paper is organized as follows. The continuum mechanics approach to ceramic powder densification is presented in Sec. 3. This includes the theoretical model as well as its finite element implementation. In Sec. 4 the technique for parameter identification is described by a multi-objective optimization of simulations of experimental tests. Finally, in Sec. 5, numerical simulations are presented of industrial powder compaction processes, namely axisymmetric tablet formation and three-dimensional tile forming. Die wall friction and deformation of the mould are taken into account. Simulated densities and lateral forces on die walls are compared with experimental results.

\section{Material}

The material considered in the present study is the aluminum silicate spray dried powder manufactured by Sacmi S.C. (Imola, Italy), labelled I14730, and described in Bosi et al. [10]. Two different water contents are considered, namely, $w=5.5 \%$ and $w=7.5 \%$, corresponding to values used in the industrial forming of traditional ceramics. The granule density, obtained with an helium pycnometer [11], is $\rho_{t}=2.599 \mathrm{~g} / \mathrm{cm}^{3}$.

\section{Constitutive framework for ceramic powder densification}

\subsection{Constitutive model}

The constitutive model was originally developed for the description of the compaction of alumina powder by Bigoni and co-workers [12-16]. That model has been modified in the present work, so as to make it more suitable for the description of the compaction of aluminum silicate powder.

All the essential equations of the constitutive model are given in Box 1. For details, the interested reader is referred to the above-mentioned references. The main features of the model can be summarized as follows:

(a) Non-linear elastic law

Granular materials typically show non-linear response in the elastic regime, whereas partially and fully densified green bodies behave linearly. The elastic law adopted in the model, Eq. (2), is able to describe this transition of elastic properties.

(b) Extremely flexible yield function [so-called 'BP yield function', 17] 
Powders and dense materials are described by yield loci of remarkably different shape. The $\mathrm{BP}$ yield function has the unique feature to continuously describe a transition between yield surfaces typical of different materials.

(c) Cooper-Eaton hardening law [18]

The first hardening law, Eq. (8), describes the densification behaviour of the material subject to isotropic compression. This law is based on a micro-mechanical model originally proposed by Cooper and Eaton [18].

(d) Increase in cohesion

The compaction process of a ceramic powder gives a form to the green body, making it at the same time cohesive and tractable for subsequent processing. The second hardening law, Eq. (9), describes the increase in cohesion at increasing forming pressure.

(e) Elasto-plastic coupling

During the compaction of a ceramic powder, the elastic stiffness of the material increases, a phenomenon which is clearly visible, for instance, from the unloading curves of a simple uniaxial compaction test performed at different final forming pressures [see 14]. The elastic stiffening of a ceramic powder during densification is connected with the volumetric plastic deformation of the material and has been addressed both experimentally and computationally [19, 20]. It is accounted for by incorporating into the model the so-called 'elasto-plastic coupling' [21-24]. For a description of the concept of elasto-plastic coupling and its use to model ceramic powder compaction, see for instance Stupkiewicz et al. [25].

Compression and extension triaxial tests have been performed on aluminum silicate powder, commonly used in industrial practice to produce ceramic tiles [see 10]. These experimental results, for the first time available for aluminum silicate, indicated the necessity to introduce into the modelling a new hardening law describing the increase of deviatoric strength as related to the deviatoric plastic deformation, see Eq. (10) in Box 1.

This hardening law has been adapted from the class of isotropic hardening laws proposed in Poltronieri et al. [26] to describe the nonlinear behaviour of concrete. These hardening laws display two crucial features: (i) they can be given both in an incremental and in the corresponding finite form; (ii) they describe a smooth transition from linear elastic to plastic behaviour, incorporating linear and nonlinear hardening, and may approach the perfectly plastic limit in the latter case. In particular, all the three hardening laws adopted in the present model can be formulated in a finite form, see Eqs. (8)-(10) in Box 1, which allows for a more efficient finite element implementation.

The constitutive model is defined by 22 material parameters (see Tab. 1), so that an identification of these material parameters is needed and will be performed through a technique combining direct fitting of experimental results together with a multi-objective optimization on simulated experiments (Sec. 4). 


\section{Box 1: Constitutive equations of the model for ceramic powder compaction}

1. Additive split of strain into an elastic $\varepsilon^{e}$ and plastic $\varepsilon^{p}$ components:

$$
\varepsilon=\varepsilon^{e}+\varepsilon^{p}
$$

2. Non-linear elastic stress/strain law:

$$
\begin{aligned}
& \boldsymbol{\sigma}\left(\varepsilon_{e}, e_{v}^{p}\right)=\left\{-\frac{2}{3} \mu e_{v}^{e}+c\right. \\
& \left.+\left(p_{0}+c\right)\left[\left(d\left(e_{v}^{p}\right)-\frac{1}{d\left(e_{v}^{p}\right)}\right) \frac{\left(1+e_{0}\right) e_{v}^{e}}{\kappa}-\exp \left(-\frac{\left(1+e_{0}\right) e_{v}^{e}}{d\left(e_{v}^{p}\right)^{1 / n} \kappa}\right)\right]\right\} \boldsymbol{I}+2 \mu \boldsymbol{\varepsilon}^{e},
\end{aligned}
$$

where $\boldsymbol{\sigma}$ is the stress, $e_{v}^{e}=\operatorname{tr} \varepsilon^{e}$ and $e_{v}^{p}=\operatorname{tr} \varepsilon^{p}$ are the elastic and plastic volumetric strains, respectively, $p_{0}$ is the initial confinement and $e_{0}$ the initial void ratio.

3. Elasto-plastic coupling:

$$
d=1+B\left\langle p_{c}-p_{c b}\right\rangle, \quad \mu(d)=\mu_{0}+c\left(d-\frac{1}{d}\right) \mu_{1},
$$

4. BP yield function:

$$
F\left(\boldsymbol{\sigma}, M, p_{c}, c\right)=f\left(p, M, p_{c}, c\right)+q g(\theta),
$$

where $p=\operatorname{tr} \boldsymbol{\sigma}, q=\sqrt{3 \operatorname{dev} \boldsymbol{\sigma} \cdot \operatorname{dev} \boldsymbol{\sigma} / 2}$, and $f(p)$ and $g(\theta)$ are the meridian and deviatoric functions:

$$
\begin{gathered}
f\left(p, M, p_{c}, c\right)=-M p_{c} \sqrt{\left[\phi-\phi^{m}\right][2(1-\alpha) \phi+\alpha]}, \quad \phi=\frac{p+c}{p_{c}+c}, \\
g(\theta)=\cos \left[\beta \frac{\pi}{6}-\frac{1}{3} \cos ^{-1}(\gamma \cos 3 \theta)\right]
\end{gathered}
$$

5. Non-associative plastic flow rule:

$$
\dot{\boldsymbol{\varepsilon}}^{p}=\dot{\lambda}\left[\boldsymbol{Q}-\frac{1}{3} \epsilon(1-\phi)(\operatorname{tr} \boldsymbol{Q}) \boldsymbol{I}\right], \quad \boldsymbol{Q}=\frac{\partial F}{\partial \boldsymbol{\sigma}} .
$$

6. Hardening laws:

$$
\begin{aligned}
e_{v}^{p} & =-\frac{e_{0}}{1+e_{0}}\left\{a_{1} \exp \left(-\frac{\Lambda_{1}}{p_{c}}\right)+a_{2} \exp \left(-\frac{\Lambda_{2}}{p_{c}}\right)\right\}, \\
c & =c_{\infty}\left[1-\exp \left(-\Gamma<p_{c}-p_{c b}>\right)\right], \\
M & =M_{0}+\frac{k_{1}}{\delta} \frac{\left(1+\delta J_{2}^{p}\right)^{n-1}-1}{(n-1)\left(1+\delta J_{2}^{p}\right)^{n-1}}, \quad J_{2}=\frac{1}{2} \operatorname{dev} \varepsilon^{p} \cdot \varepsilon^{p} .
\end{aligned}
$$




\subsection{Finite Element implementation and integration of the material model into commercial FEM codes}

The most efficient way to implement an elastoplastic constitutive model, to be used with commercial Finite Element software, is to develop an external subroutine describing the material response.

This procedure can be carried out in Abaqus FEA by coding a UMAT (User MATerial) subroutine, which interfaces with the FE software through a standardized parameter list in the subroutine call statement. The UMAT subroutine is compiled and linked to the main Abaqus executable prior to the job execution.

The implementation of the constitutive model for ceramic powder compaction sketched in Box 1 must overcome non-standard difficulties, which include nonlinear elastic behavior, even at small strain, and elastoplastic coupling. Furthermore, the 'stretchable' pressure-sensitive yield function introduced by Bigoni and Piccolroaz [17] has the inconvenience that, in order to be convex, must be defined $+\infty$ in some regions outside the elastic domain. This fact, which prevents the application of standard return-mapping techniques for the solution of the plasticity equations, has been recently overcome by Brannon and Leelavanichkul [27], Penasa et al. [15], and Stupkiewicz et al. [25], using different strategies.

The last-mentioned technique, based on a implicit definition of the BP yield function, has been used in the current implementation of the constitutive elastoplastic model. In order to increase the stability and robustness of the subroutine, a fully-implicit return mapping technique has been combined with a substepping procedure [28, 29].

The development of the UMAT subroutine code $^{1}$ has been carried out by using the advanced hybrid symbolic-numeric approach implemented in AceGen [30, 31], a symbolic code generator available as a package of Wolfram Mathematica. The combination of automatic differentiation (AD) technique, optimization of formulae and automatic generation of computer code (such as $\mathrm{C}++$, FORTRAN and Matlab) available in AceGen made possible to efficiently and rapidly prototype the new numerical procedure and benchmark the generated code, which can also be tested within the Mathematica environment by means of the flexible FE code AceFEM.

\section{Material parameter identification by simulation of experimental tests}

\subsection{Experimental tests on aluminum silicate powder}

Some of the material parameters, involved in the constitutive model for ceramic powder densification, were identified directly from the results of a set of experimental tests by Bosi et al. [10]. In particular, the following tests were performed:

(a) Uniaxial deformation test were carried out by imposing compaction of the aluminum silicate powder in a $30 \mathrm{~mm}$ diameter mould, filled until a height of $4 \mathrm{~mm}$. After reaching the desired pressure $\sigma_{1}=\{5,10,30,45,60,80\} \mathrm{MPa}$, the green body was unloaded and extracted from the device.

\footnotetext{
${ }^{1}$ The developed code has been also translated into a USERMAT subroutine for the use in the Ansys environment.
} 
The uniaxial compaction test provided the force-displacement curves, from which the compaction behaviour of the powder, i.e. the relation between forming pressure and density, was deduced. This allowed the calibration of the first hardening law, Eq. (8), and the related material parameters, $a_{1}, \Lambda_{1}, a_{2}, \Lambda_{2}$. However, the identification of these parameters by a uniaxial compaction test is affected by the fact that the state of stress and deformation is not purely isotropic. This has required an adjustment of the values identified by Bosi et al. [10], as explained in Sec. 4.4.

The logarithmic elastic bulk modulus $\kappa$ has been evaluated from the linear elastic phase (at very low pressure, prior to the breakpoint pressure $p_{c b}$ ).

(b) Equi-biaxial flexure tests were performed on the green body tablets produced in the cylindrical mould, following the ASTM C 1499-05 'Standard Test Method for Monotonic Equi-biaxial Flexural Strength of Advanced Ceramics at Ambient Temperature'. This allowed the calibration of the second hardening law, Eq. (9), and the related material parameters, $c_{\infty}, \Gamma, p_{c b}$, describing the increase of cohesion with forming pressure.

(c) Compression and extension triaxial tests have been performed on pre-compacted (at $\sigma_{1}=40$ $\mathrm{MPa}$ ) cylindrical specimens (38.2 $\mathrm{mm}$ diameter and $70 \mathrm{~mm}$ height), according to the ASTM D 2664 95a 'Standard Test Method for Triaxial Compressive Strength of Undrained Rock Core Specimens Without Pore Pressure Measurements'. The results of these tests have been used together with the results of uniaxial compaction tests in the optimization procedure.

All the other material parameters, not identified directly through the mechanical tests, are determined by simulating the experiments and performing an iterative multi-objective optimization, which was carried out with simplified numerical models, involving a small number of finite elements and neglecting the effects of friction. This approach significantly speeded up the FE simulations, so that the entire parameter identification could be performed on a simple laptop computer in a reasonable computational time. This procedure leads to very accurate results, in particular for the uniaxial deformation tests, which are considered with great interest for effective simulation of industrial tile forming processes.

In both uniaxial deformation and triaxial test simulations, the FE analyses were carried out in the Abaqus FEA environment using axisymmetric 8-node biquadratic elements (CAX8).

\subsection{Simulation of uniaxial deformation tests}

The numerical simulation of uniaxial deformation tests, as performed by Bosi et al. [10], involves the execution of the following four steps:

1. Geostatic step: As the powder before compaction is cohesionless, the analysis starts assuming a small confinement given by an initial value of isotropic stress, $p_{0}$, which is equilibrated by an equivalent external load in a geostatic step. The initial values of isotropic stress and void ratio used in the simulation are $p_{0}=0.9 \mathrm{MPa}$ and $e_{0}=2.04$.

2. Loading: In this step a uniform pressure is applied to the upper face of the sample, see Fig. 1, while the constraints at the bottom and lateral surfaces reproduce frictionless contact with the mould. 
3. Unloading: In this phase the pressure on the upper face is removed, while the boundary constraints at bottom and lateral faces are kept active, so as to simulate the removal of the punch.

4. Extraction: The supports on the right side of the sample are deactivated in the last step, so that the compact is free to expand transversally, which reproduces ejection from the mould.

Figure 1 shows the undeformed mesh with the constraints to reproduce uniaxial deformation conditions (left) and the deformed mesh at the end of the loading step (right, the contours denote vertical displacement).
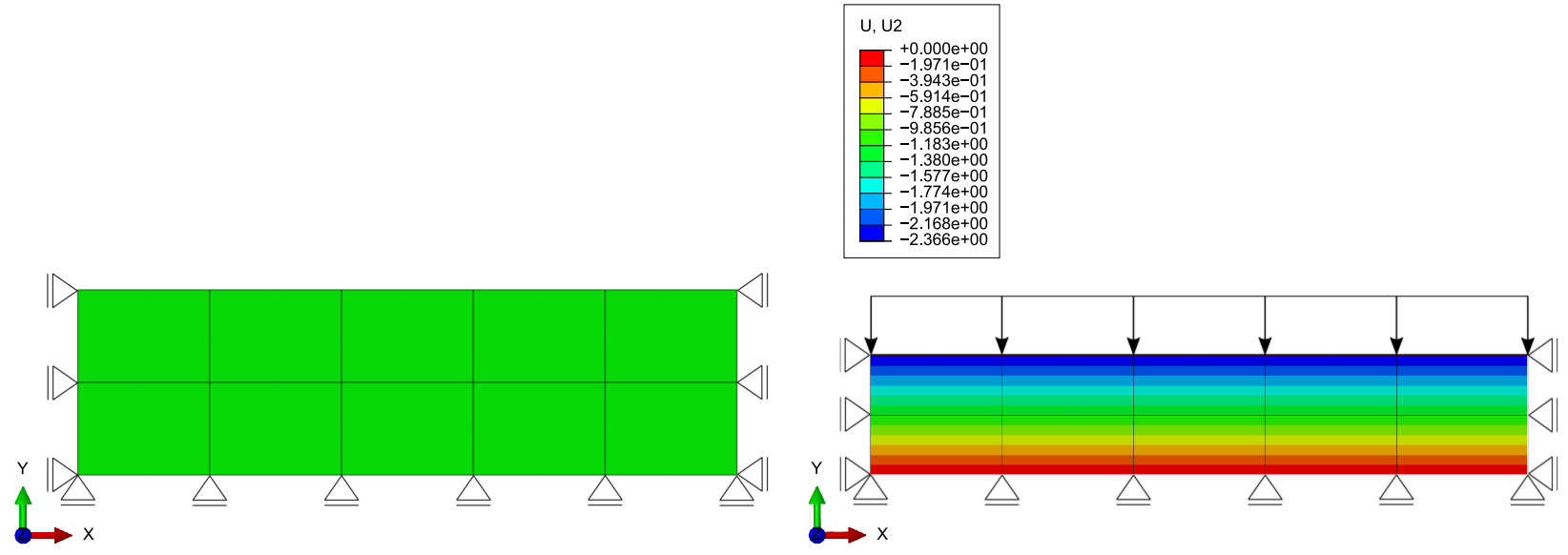

Figure 1: Simplified FE model for uniaxial compaction simulation. Undeformed mesh (left) and deformed mesh at the end of loading step (right, contours denote vertical displacement).

\subsection{Simulation of compression and extension triaxial tests}

The FE analysis was carried out to simulate the triaxial tests performed by Bosi et al. [10], on cylindrical samples, pre-compacted at $40 \mathrm{MPa}$. This involves two stages. The first stage corresponds to the preparation of cylindrical specimens, formed by uniaxial compaction at $40 \mathrm{MPa}$, followed by an isotropic compaction at $40 \mathrm{MPa}$ in the triaxial cell. After unloading, the second stage corresponds to the actual triaxial test. Both compression and extension triaxial tests were simulated in the way described below.

\section{Stage 1: forming of cylindrical specimens}

1. Geostatic step: The first geostatic step aims to equilibrate, by imposing an external pressure, $p_{0}$, the assumed initial isotropic confinement in the ceramic powder. The initial values of isotropic stress and void ratio used in the simulation are $p_{0}=0.9 \mathrm{MPa}$ and $e_{0}=2.04$.

2. Uniaxial compaction at $\sigma_{2}=40 \mathrm{MPa}$ : The powder is first compacted in a uniaxial deformation step at a final vertical stress equal to $40 \mathrm{MPa}$. 
3. Unloading: In this step the sample is unloaded.

4. Isotropic compaction at $p=40 \mathrm{MPa}$ : An uniform pressure of $40 \mathrm{MPa}$ is applied on both faces of the sample (see Fig. 2): $\sigma_{1}=\sigma_{2}=40 \mathrm{MPa}$.

5. Unloading: In this step the sample is unloaded.

\section{Stage 2: triaxial test}

1. Isotropic loading: The confinement pressure is applied on the faces of the sample: $\sigma_{1}=$ $\sigma_{2}=\{2,5,10,15,20,30\} \mathrm{MPa}$.

2. Deviatoric loading: Compression triaxial test: on the upper face of the cylinder a negative displacement is imposed, so as to reduce the hight of the sample. Extension triaxial test: on the lateral face of the cylinder a negative displacement is imposed, so as to reduce the width of the sample.

Figure 2 shows the undeformed mesh with the constraints to reproduce uniaxial compaction conditions (step 2 of Stage 1, left), the deformed mesh at the end of forming of the cylindrical specimen (end of Stage 1, centre), the deformed mesh at the end of triaxial test (right). In the central and right figures, contours denote vertical displacement.
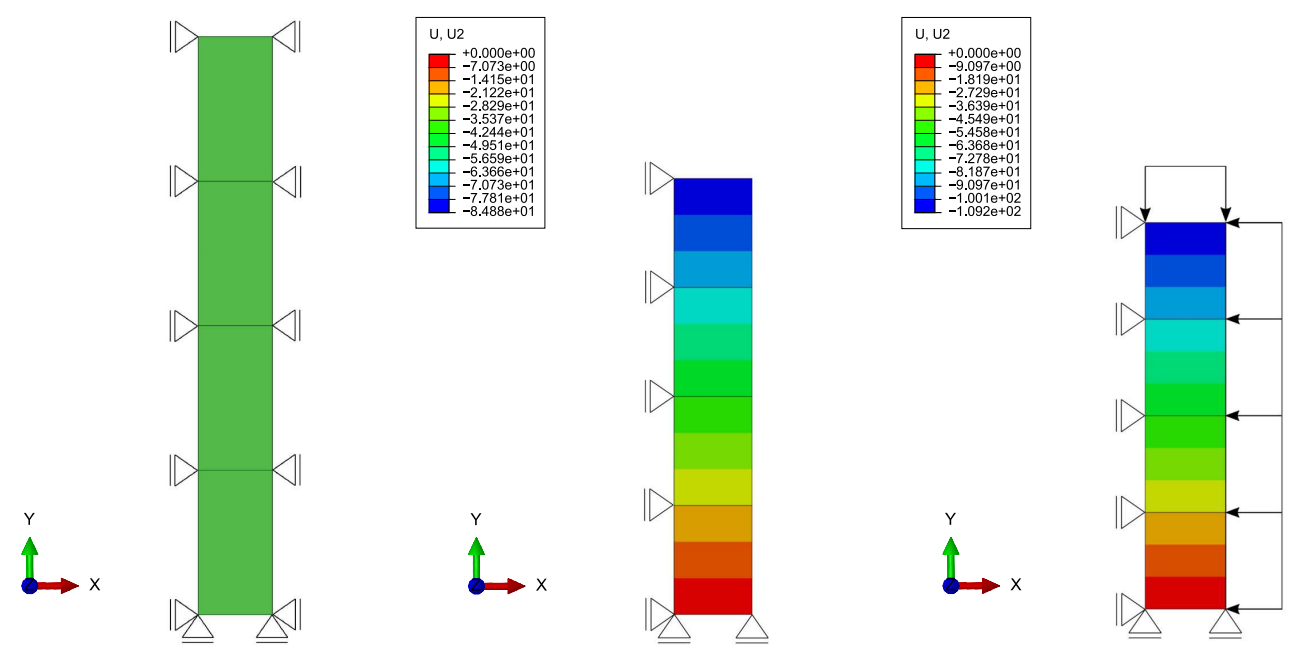

Figure 2: Simplified FE model for triaxial test simulation. Undeformed mesh (left), deformed mesh at the end of Stage 1 (forming of cylindrical specimen, centre) and deformed mesh at the end of Stage 2 (triaxial test, right). The contours denote vertical displacement.

\subsection{Material parameter identification by multi-objective optimization}

A multi-objective optimization procedure has been performed in order to identify the constitutive parameters not directly calibrated from the experimental tests performed by Bosi et al. [10]. 
These parameters include: the elasto-plastic coupling parameters, $B, n, \mu_{0}$ and $\mu_{1}$, governing the evolution of elastic properties with plastic deformation, Eq. (3); the parameters involved in the deviatoric hardening rule, Eq. (10), $M_{0}, k_{1}, \delta_{1}$ and $n_{1}$; the parameter $\epsilon$ defining the non-associativity, Eq. (7).

In addition, the parameters $a_{1}, \Lambda_{1}, a_{2}, \Lambda_{2}$, governing the pressure-density behaviour of the powder in isotropic compression, were included in the optimization. This was done because isotropic compression tests are not available, and thus the values identified by Bosi et al. [10] through uniaxial compaction tests require an adjustment.

With the introduction of the deviatoric hardening rule, Eq. (10), the parameter $M$, describing the pressure-sensitivity, Eq. (5), evolves during the densification. This fact is expected to influence also the other parameters governing the meridian shape of the BP yield function, $m$ and $\alpha$, which are thus also included in the optimization.

The multi-objective optimization has been performed by employing the algorithms available in the Dakota Framework [32], which allows the optimization with both gradient and nongradient-based methods. The chosen optimization strategy aims to find the best possible fit between simulated results and experimental curves, both for uniaxial compression and triaxial compression tests.

The random combination of material parameters, even inside their allowable ranges, may lead to unconsistent results or lack of convergence. For this reason a gradient-free approach has been preferred in the optimization procedure. As a sufficiently precise starting point was not available, both global and local optimization methods have been used to efficiently estimate the material parameters.

The 'hybrid' procedure involves first a Pareto optimization, by means of moga (multi-objective genetic algorithm). After a sufficiently high number of iterations of the global algorithm, the best five solutions are refined by a local optimization method (pattern-search). The convergence of the optimization strategy, in terms of relative error as a function of the number of iterations, is shown in Fig. 3.
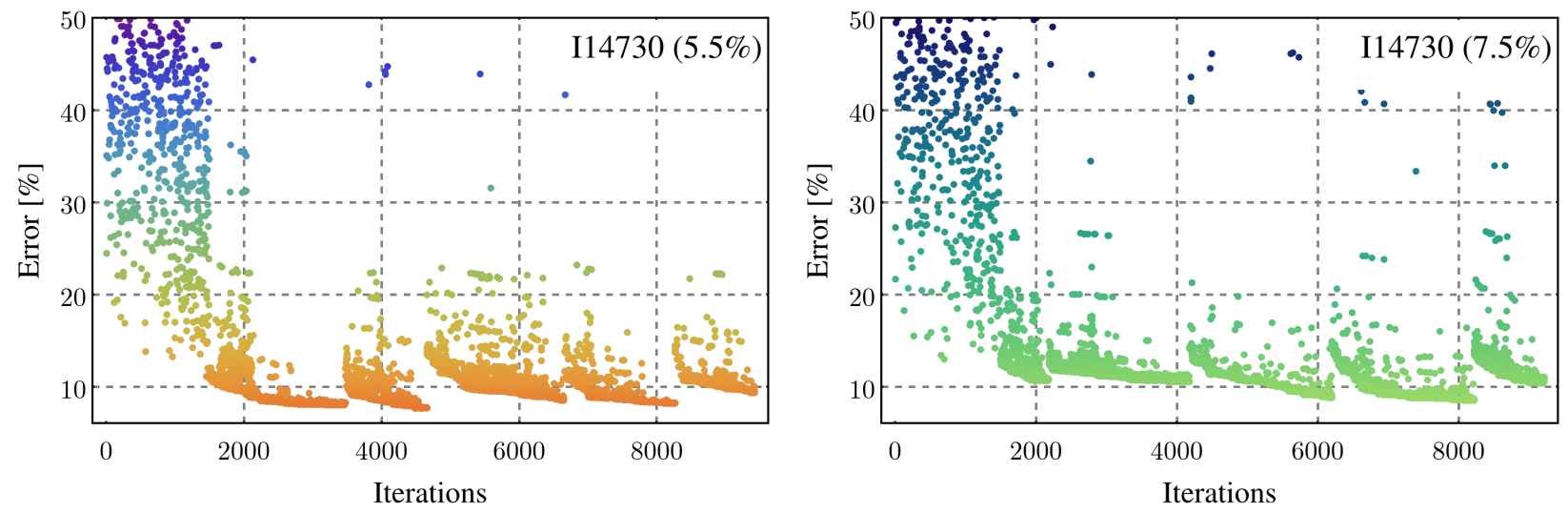

Figure 3: Convergence of the hybrid optimization algorithm for the material parameter identification of aluminum silicate powder with $w=5.5 \%$ (left) and $w=7.5 \%$ (right) water content.

The graph shows how, with the increase of the number of iterations, the parameters generating low error tend to become more dense (moga algorithm). After 1500 iterations, the graph shows the 
convergence of the local optimization algorithm (pattern-search) for the five best solutions of the global algorithm.

In order to reduce the size of the problem, the optimization focused on reaching the best fit of the experimental tests considered of highest industrial interest, namely:

- Uniaxial compaction tests at $\sigma_{2}=\{45,60\} \mathrm{MPa}$

- Triaxial compression tests at cell confinement: $\sigma_{1}=\sigma_{3}=\{20,30\} \mathrm{MPa}$

The final set of parameters for the compaction of aluminum silicate for two different water contents, $w=5.5 \%$ and $w=7.5 \%$ are reported in Tab. 1 .

With the identified parameters, it is possible to obtain a good agreement between numerical and experimental results also in tests which are not considered in the optimization. This is an indication of the consistency of the constitutive model and of the validity of the optimization procedure. Figure 4 shows the uniaxial compaction (force vs. displacement) curves for water content $w=5.5 \%$ (left) and $w=7.5 \%$ (right). Figure 5 shows the compression/extension triaxial (von Mises stress vs. axial strain) curves for water content $w=5.5 \%$ (left) and $w=7.5 \%$ (right). 
Table 1: Material parameters for the compaction of aluminum silicate I14730, for two different water contents, $w=5.5 \%$ and $w=7.5 \%$. Seven parameters were obtained directly from the experiments by Bosi et al. [10], the other 15 parameters have been identified by multi-objective optimization.

\begin{tabular}{|c|c|c|c|c|}
\hline & \multirow{2}{*}{\multicolumn{2}{|c|}{ Parameter }} & \multicolumn{2}{|c|}{ Aluminium Silicate I14730 } \\
\hline & & & $w=5.5 \%$ & $w=7.5 \%$ \\
\hline \multicolumn{5}{|c|}{ Parameters identified directly from experiments } \\
\hline Log. bulk modulus & $(1)$ & $\kappa$ & 0.08 & 0.099 \\
\hline \multirow{3}{*}{ Yield surface } & $(2)$ & $p_{\mathrm{c} 0}$ & $0.09 \mathrm{MPa}$ & $0.09 \mathrm{MPa}$ \\
\hline & $(3)$ & $\beta$ & 0.1 & 0.08 \\
\hline & $(4)$ & $\gamma$ & 0.9 & 0.9 \\
\hline \multirow{3}{*}{ Hardening law (9) } & $(5)$ & $p_{\mathrm{cb}}$ & $0.22 \mathrm{MPa}$ & $0.17 \mathrm{MPa}$ \\
\hline & $(6)$ & $c_{\infty}$ & $1.10 \mathrm{MPa}$ & $1.35 \mathrm{MPa}$ \\
\hline & $(7)$ & $\Gamma$ & $0.06 \mathrm{MPa}^{-1}$ & $0.10 \mathrm{MPa}^{-1}$ \\
\hline \multicolumn{5}{|c|}{ Parameters identified by multi-objective optimization } \\
\hline \multirow{3}{*}{ Yield surface } & $(8)$ & $M_{0}$ & 0.398 & 0.506 \\
\hline & (9) & $m$ & 2.26 & 3.17 \\
\hline & $(10)$ & $\alpha$ & 1.09 & 1.367 \\
\hline \multirow{4}{*}{ Hardening law (8) } & $(11)$ & $a_{1}$ & 0.763 & 0.780 \\
\hline & $(12)$ & $\Lambda_{1}$ & $0.702 \mathrm{MPa}$ & $0.507 \mathrm{MPa}$ \\
\hline & $(13)$ & $a_{2}$ & 0.154 & 0.154 \\
\hline & $(14)$ & $\Lambda_{2}$ & $36.285 \mathrm{MPa}$ & $25.19 \mathrm{MPa}$ \\
\hline \multirow{3}{*}{ Hardening law (10) } & $(15)$ & $k_{1}$ & 301.417 & 30.08 \\
\hline & $(16)$ & $\delta_{1}$ & 456.806 & 165.405 \\
\hline & $(17)$ & $n_{1}$ & 3.647 & 9.149 \\
\hline \multirow{4}{*}{ E-P coupling $(3)$} & $(18)$ & $B$ & $9.580 \mathrm{MPa}^{-1}$ & $6.908 \mathrm{MPa}^{-1}$ \\
\hline & (19) & $n$ & 11.949 & 11.749 \\
\hline & $(20)$ & $\mu_{0}$ & $0.223 \mathrm{MPa}$ & $9.822 \mathrm{MPa}$ \\
\hline & $(21)$ & $\mu_{1}$ & 24.678 & 5.269 \\
\hline Plastic flow (7) & $(22)$ & $\varepsilon$ & 0.916 & 0.586 \\
\hline
\end{tabular}



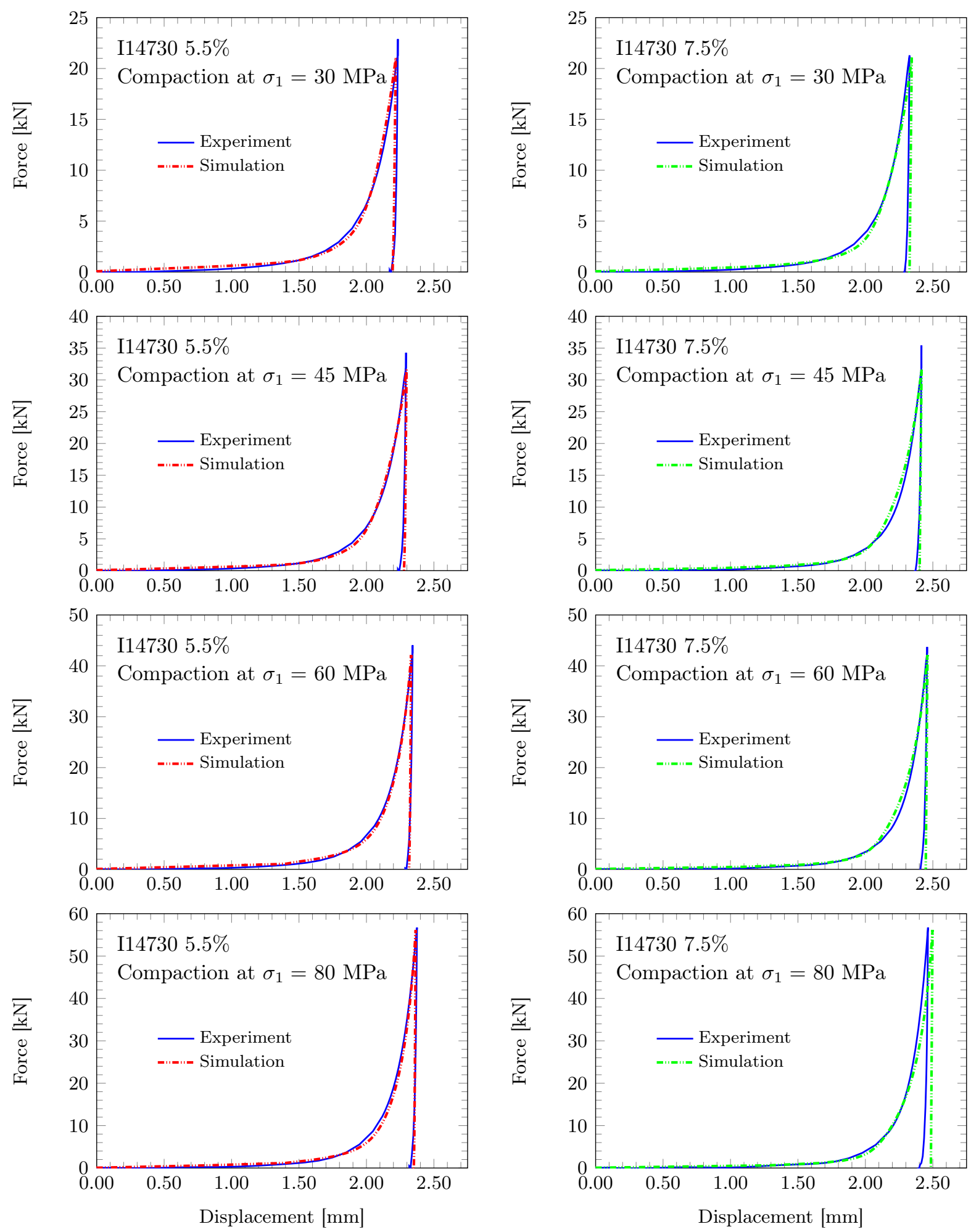

Figure 4: Uniaxial compaction test: Comparison between experimental results and numerical simulations obtained with the identified material parameters (Tab. 1), for water content $w=5.5 \%$ (left) and $w=7.5 \%$ (right). The experimental curves corresponding to forming at $\sigma_{2}=45$ and $60 \mathrm{MPa}$ were used in the optimization procedure. 

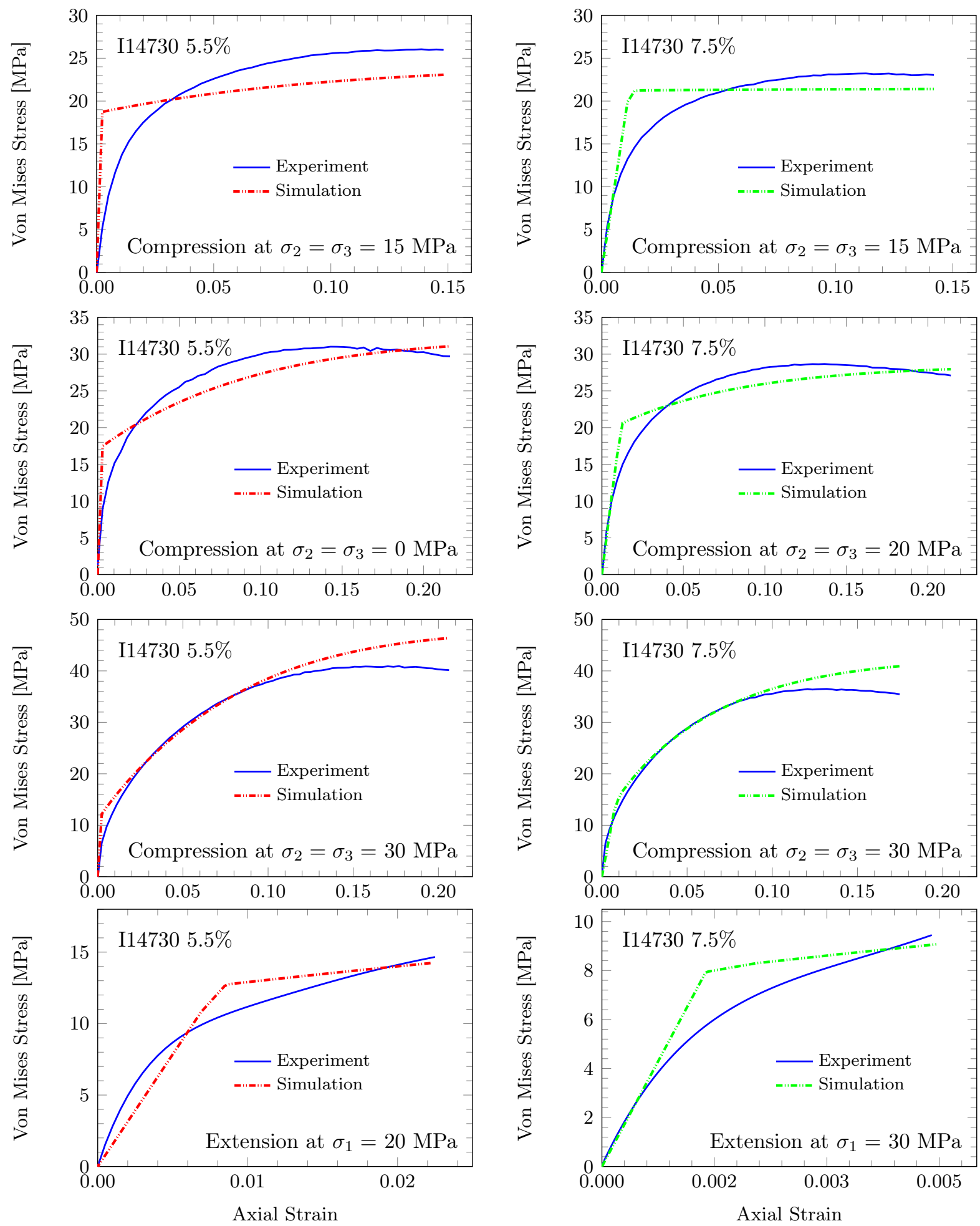

Figure 5: Compression/extension triaxial test: Comparison between experimental results and numerical simulations obtained with the identified material parameters (Tab. 1), for water content $w=5.5 \%$ (left) and $w=7.5 \%$ (right). The experimental curves corresponding to compression tests at confinement pressure $\sigma_{1}=\sigma_{3}=20$ and $30 \mathrm{MPa}$ were used in the optimization procedure. 


\section{Numerical simulation of industrial powder compaction processes}

\subsection{Experimental identification of friction coefficient between powder and die wall}

At the end of process of powder compaction, the sample is unloaded and extracted from the forming device. In this phase, friction between the green body and the steel matrix requires the application of an axial force on the tablet to complete the extraction. In the experimental tests performed by Bosi et al. [10], it was possible to measure the tangential force $T$ required to remove the green body from the mould. The normal force $N$ on the steel matrix can be calculated from the measured radial deformation of the sample after extraction and the elastic moduli, measured by means of ultrasound technique by Argani et al. [19].

The static friction coefficient can be estimated from the relationship between tangential and normal forces acting on the die wall. The calculation of static friction coefficient for forming pressure of $45 \mathrm{MPa}$ yielded $\mu_{s}=T / N=0.18$. This value has been used in the following FE simulations in order to accurately reproduce the contact interaction between ceramic powder and steel matrix.

\subsection{Numerical simulation of axisymmetric tablet forming}

Finite element analyses, involving contact interaction and friction, have been performed to accurately reproduce the uniaxial compaction tests performed by Bosi et al. [10]. In these simulations, the complete forming device, composed by matrix, upper and lower punches, was modelled, and the interaction between each single part and the ceramic powder was taken into account.

The experimental set-up used in the uniaxial compaction test of I14730 aluminum silicate powder, performed by Bosi et al. [10] is shown in Fig. 6: the schematic cross section on the left and a photograph in the centre. The elements considered in the simulation are shown on the right: ceramic powder, cylindrical matrix, bottom and upper punches.

The simulation comprises the following steps: geostatic step, in which an initial confinement $p_{0}$ is imposed on the powder; uniaxial compaction phase, in which the upper punch is loaded by the given vertical pressure; unloading step, in which the load on the upper punch is removed; extraction, in which the matrix is removed in order to simulate the removal of the tablet from the cylindrical mould.

The stresses developing in the ceramic powder as well as in the mould are shown in Fig. 7. The analysis made also possible to investigate the influence of friction in the forming process and to determine the transversal pressure on the steel matrix.

The dimensions and densities of the formed tablets are reported in Tables 2 and 3, for aluminum silicate powder with water content $5.5 \%$ and $7.5 \%$, respectively. The simulation results are in good agreement with the experimental values. We note that a reduced radial 'springback' after extraction of the tablet from the mould was predicted by the numerical simulations.

A comparison of experimental and simulated compact densities is shown in Fig. 8, for water content $5.5 \%$ (left) and $7.5 \%$ (right). 

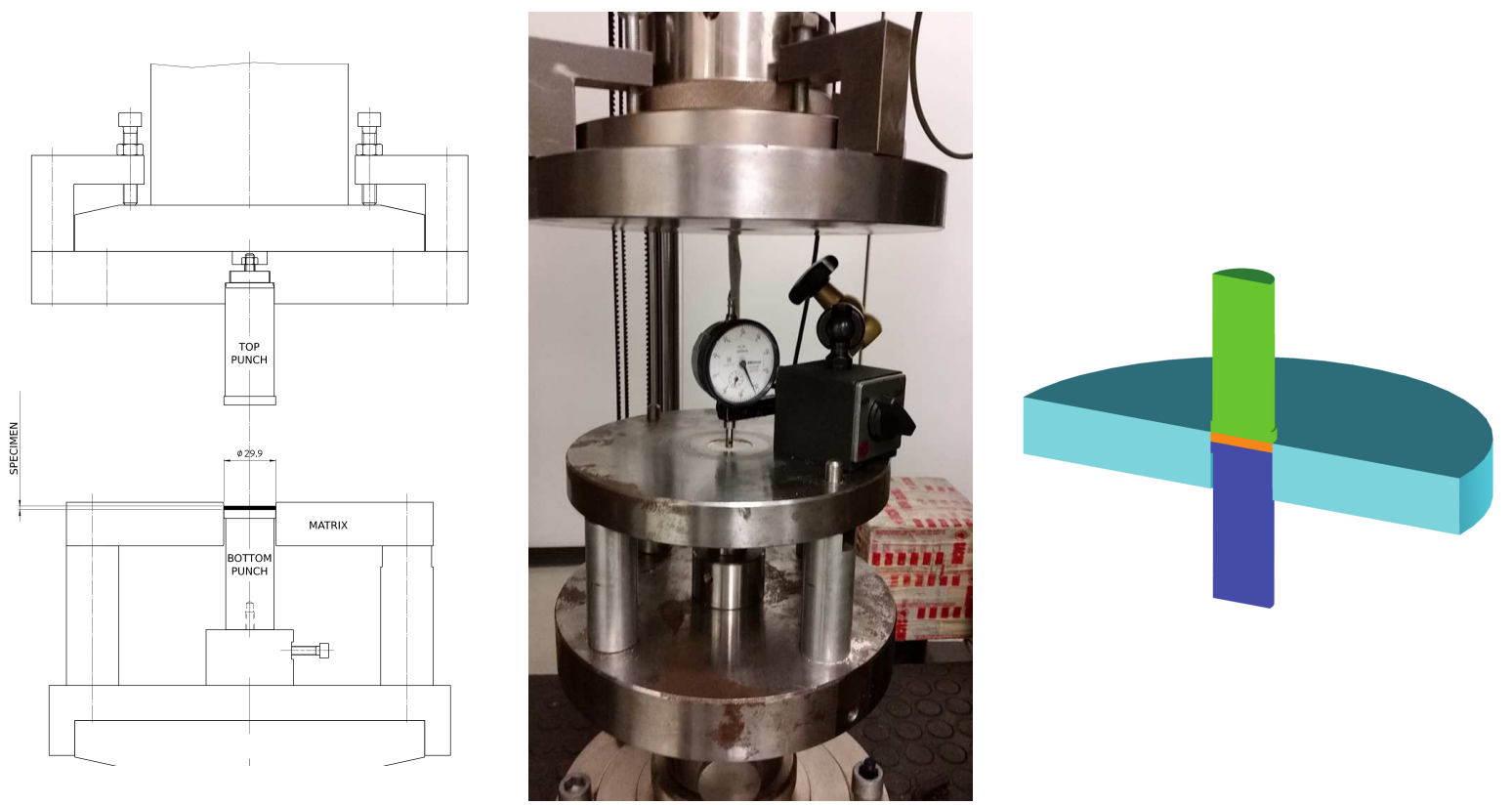

Figure 6: Experimental set-up used in the uniaxial compaction test of I14730 aluminum silicate powder, performed by Bosi et al. [10]. Cross section (left) and photograph (centre) of the forming device. The elements considered in the simulation: matrix, upper and lower punches, and ceramic powder (right). 


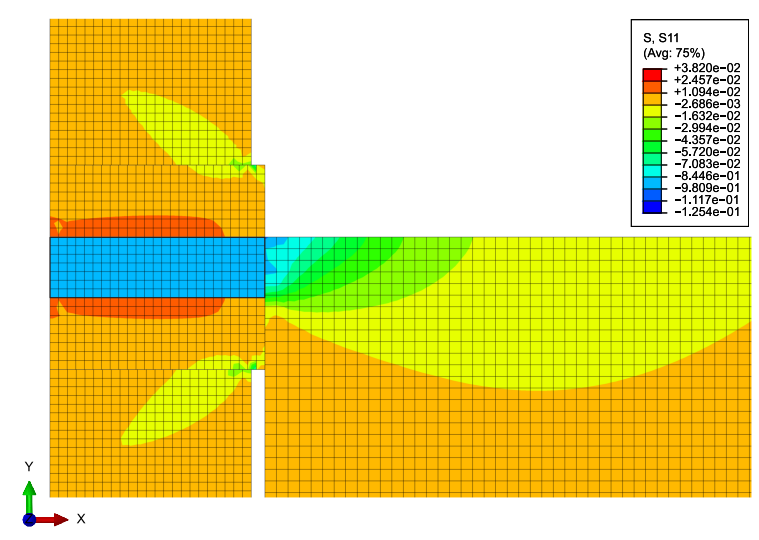

(a) Geostatic step: $\sigma_{x x}$

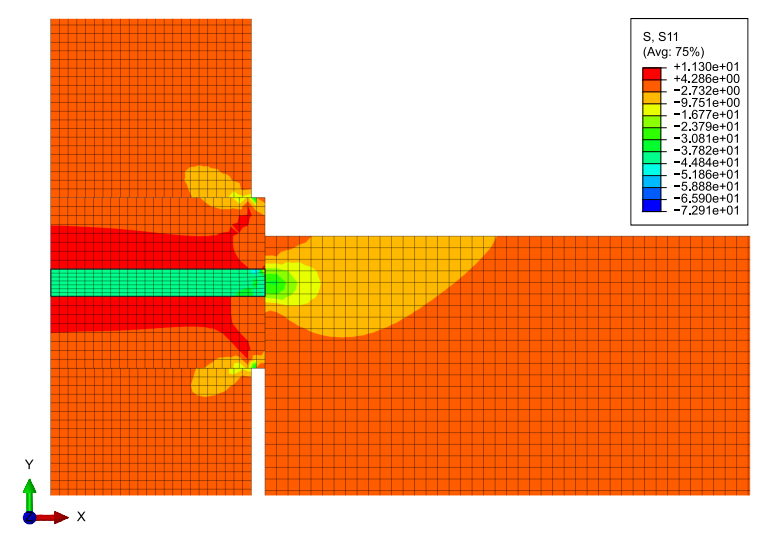

(c) End of compaction phase: $\sigma_{x x}$

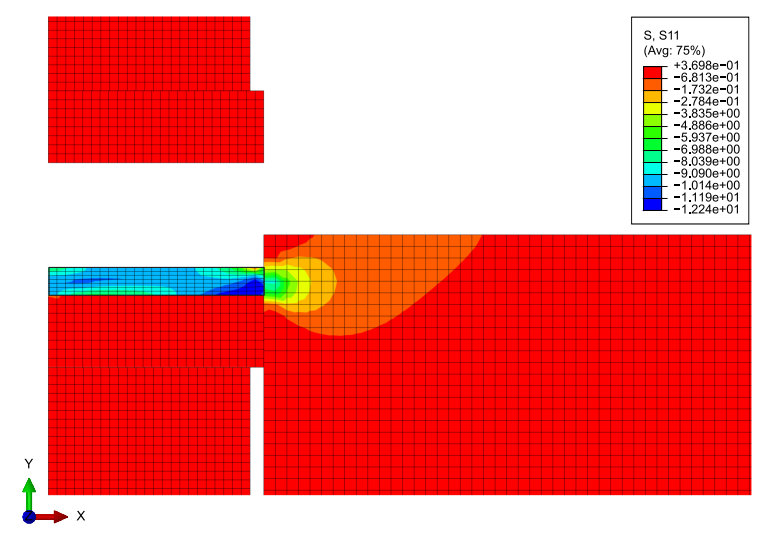

(e) Unloading: $\sigma_{x x}$

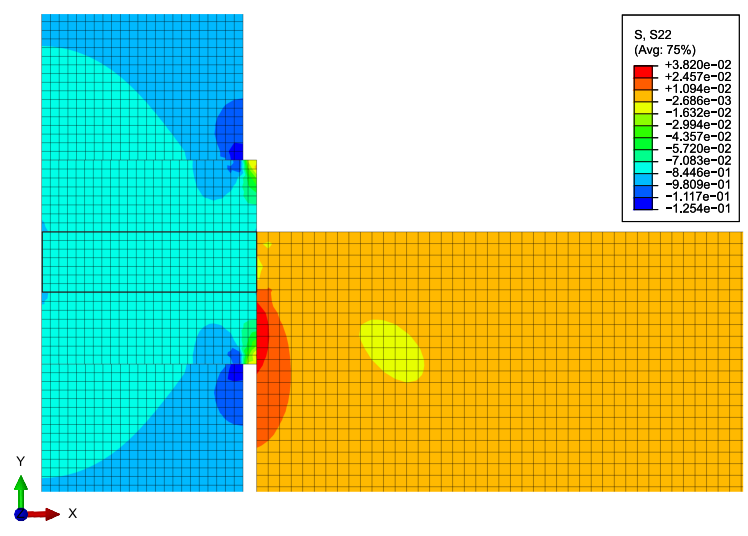

(b) Geostatic step: $\sigma_{y y}$

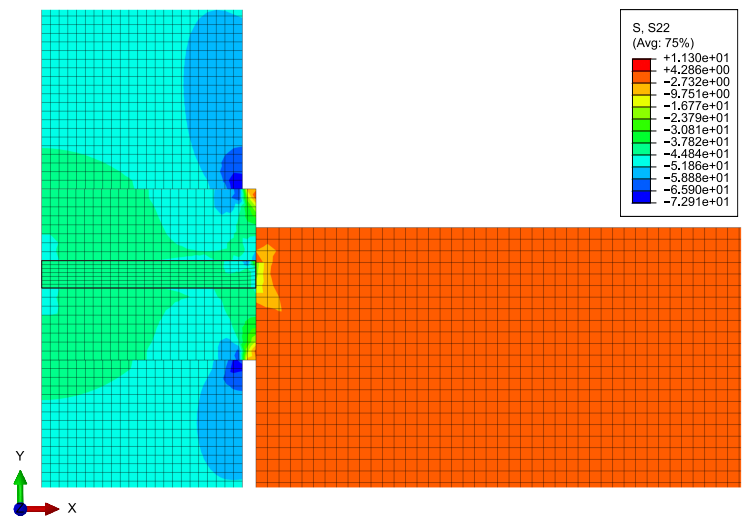

(d) End of compaction phase: $\sigma_{y y}$
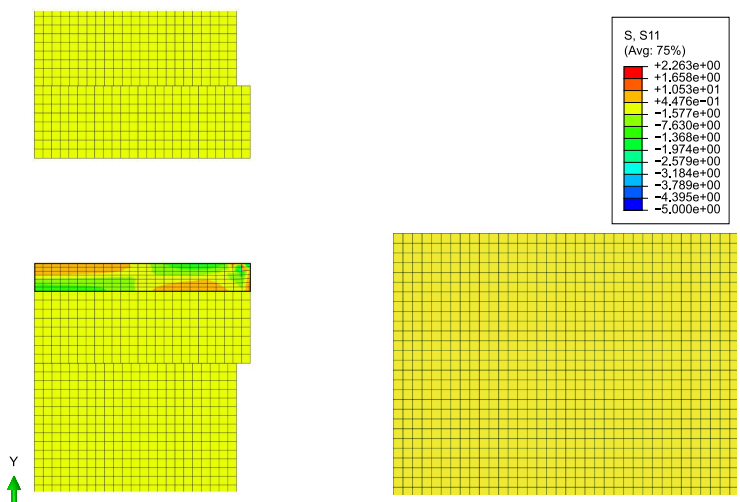

(f) Extraction of the green body: $\sigma_{x x}$

Figure 7: Axisymmetric numerical simulation of tablet forming. Stress distribution (lateral stress $\sigma_{x x}$ and axial stress $\sigma_{y y}$ ) in the ceramic powder and in the mould (composed by matrix, upper and lower punches) at the end of the main stages of powder compaction: geostatic step (upper part), axial loading (central part), axial unloading (lower part, left) and extraction of the tablet (lower part, right). 
Table 2: Dimensions and densities of I14730 aluminum silicate tablets $(\mathrm{w}=5.5 \%)$ after completion of the uniaxial compaction process and extraction from the cylindrical mould.

\begin{tabular}{cccccc}
\hline $\begin{array}{c}\text { Forming } \\
\text { pressure }[\mathrm{MPa}]\end{array}$ & $\begin{array}{c}\text { Area } \\
{\left[\mathrm{mm}^{2}\right]}\end{array}$ & $\begin{array}{c}\text { Height } \\
{[\mathrm{mm}]}\end{array}$ & $\begin{array}{c}\text { Diameter } \\
{[\mathrm{mm}]}\end{array}$ & $\begin{array}{c}\text { Density } \\
{\left[\mathrm{g} \mathrm{cm}^{-3}\right]}\end{array}$ \\
\hline \multirow{2}{*}{5} & Experiment & 706.387 & 2.338 & 29.990 & 1.653 \\
& Simulation & 708.452 & 2.392 & 30.034 & 1.611 \\
\hline \multirow{2}{*}{10} & Experiment & 706.858 & 2.198 & 30.000 & 1.757 \\
& Simulation & 705.841 & 2.225 & 29.978 & 1.738 \\
\hline \multirow{2}{*}{30} & Experiment & 708.273 & 2.042 & 30.030 & 1.957 \\
& Simulation & 703.554 & 2.004 & 29.930 & 2.007 \\
\hline \multirow{2}{*}{45} & Experiment & 708.745 & 1.966 & 30.040 & 2.009 \\
& Simulation & 703.225 & 1.923 & 29.923 & 2.071 \\
\hline \multirow{2}{*}{60} & Experiment & 708.745 & 1.880 & 30.040 & 2.056 \\
& Simulation & 703.065 & 1.865 & 29.919 & 2.089 \\
\hline \multirow{2}{*}{80} & Experiment & 708.745 & 1.844 & 30.040 & 2.081 \\
& Simulation & 702.962 & 1.817 & 29.917 & 2.130 \\
\hline
\end{tabular}

Table 3: Dimensions and densities of I14730 aluminum silicate tablets $(\mathrm{w}=7.5 \%)$ after completion of the uniaxial compaction process and extraction from the cylindrical mould.

\begin{tabular}{cccccc}
\hline $\begin{array}{c}\text { Forming } \\
\text { pressure }[\mathrm{MPa}]\end{array}$ & & $\begin{array}{c}\text { Area } \\
{\left[\mathrm{mm}^{2}\right]}\end{array}$ & $\begin{array}{c}\text { Height } \\
{[\mathrm{mm}]}\end{array}$ & $\begin{array}{c}\text { Diameter } \\
{[\mathrm{mm}]}\end{array}$ & $\begin{array}{c}\text { Density } \\
{\left[\mathrm{g} \mathrm{cm}^{-3}\right]}\end{array}$ \\
\hline \multirow{2}{*}{5} & Experiment & 705.916 & 2.236 & 29.980 & 1.730 \\
& Simulation & 704.175 & 2.221 & 29.943 & 1.746 \\
\hline \multirow{2}{*}{10} & Experiment & 705.916 & 2.074 & 29.980 & 1.865 \\
& Simulation & 703.451 & 2.089 & 29.928 & 1.858 \\
\hline \multirow{2}{*}{30} & Experiment & 707.801 & 1.918 & 30.020 & 2.011 \\
& Simulation & 702.981 & 1.870 & 29.918 & 2.077 \\
\hline \multirow{2}{*}{45} & Experiment & 707.943 & 1.836 & 30.023 & 2.085 \\
& Simulation & 702.905 & 1.795 & 29.916 & 2.148 \\
\hline \multirow{2}{*}{60} & Experiment & 708.745 & 1.772 & 30.040 & 2.102 \\
& Simulation & 702.868 & 1.740 & 29.915 & 2.159 \\
\hline \multirow{2}{*}{80} & Experiment & 708.273 & 1.814 & 30.030 & 2.148 \\
& Simulation & 702.868 & 1.711 & 29.915 & 2.295 \\
\hline
\end{tabular}



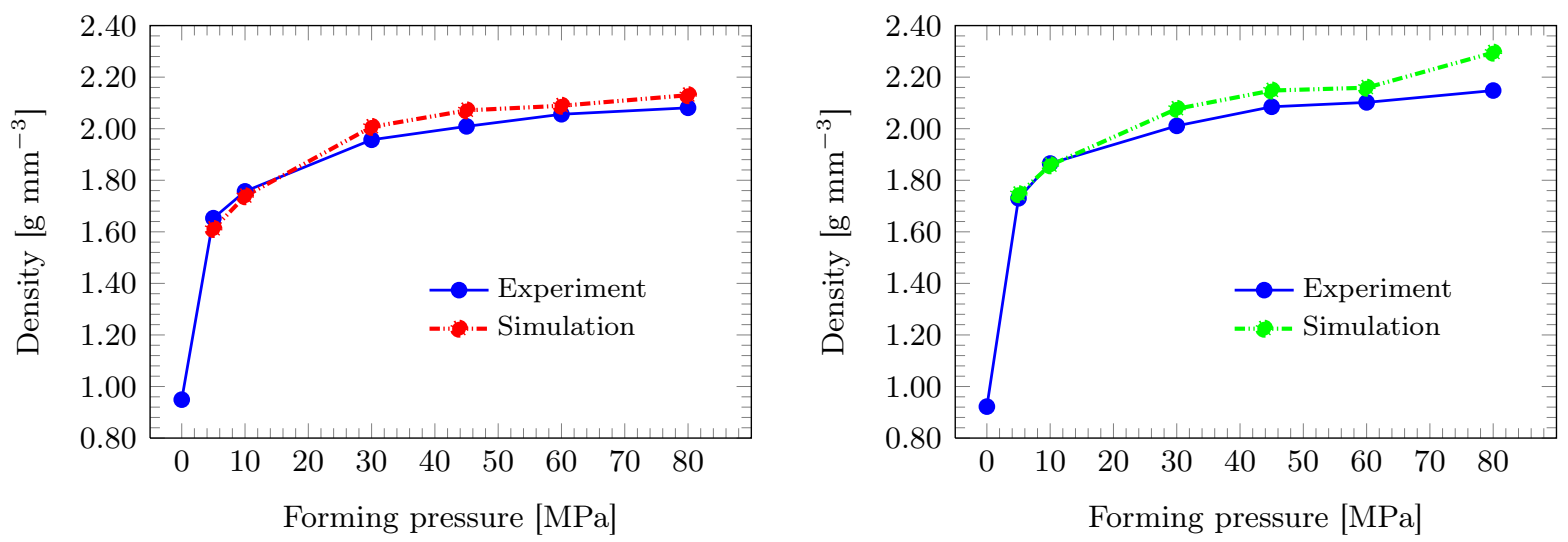

Figure 8: Densities of I14730 aluminium silicate tablets, with $5.5 \%$ and $7.5 \%$ of water content: comparison between experimental result and numerical simulation for different forming pressures. The first experimental point shows the average bulk density of the ceramic powder when inserted in the cylindrical mould.

\subsection{Three-dimensional numerical simulation of an industrial tile forming pro- cess}

Three-dimensional FE simulations of an industrial tile forming process have been performed in Abaqus FEA environment using the developed constitutive model and the identified parameters listed in Tab. 1. The 3D model involves contact interactions between ceramic powder, steel matrix and top/bottom steel plates, where the friction coefficient reported in Sec. 5.1 has been used.

Figure 9 shows the vertical and transverse stress distributions in the ceramic powder and in the mould (composed by matrix, upper and lower plates) at the end of the main stages of powder compaction.

The initial values of isotropic stress and void ratio prescribed in the geostatic step are $p_{0}=0.9$ $\mathrm{MPa}$ and $e_{0}=2.04$, respectively, which are the same values used in the axisymmetric case. This step corresponds to the initial confinement inside the mould, see Fig. 9a and 9b.

At the end of the compaction phase, Fig. $9 \mathrm{c}$ and $9 \mathrm{~d}$, we notice a non-uniform stress distribution, especially for the transversal stress $\sigma_{y y}$, in the external part of the tile, which is in contact with the die wall. This effect is mainly due to friction between the ceramic powder and the mould.

After the unloading step (removal of the upper plate), see Fig. 9e, the transversal stress $\sigma_{y y}$ in the green tile is still quite high, approximately $8 \mathrm{MPa}$, due to the lateral constraint given by the matrix.

The residual stresses at the end of the extraction phase, predicted by the finite element simulation, are shown in Fig. 9f. We notice a compressive transversal stress $\sigma_{y y}$ at the upper edge of the tile, which is balanced by a tensile $\sigma_{y y}$ at the lower edge. The capabilities of the model to predict residual stresses are crucial, since residual stresses may lead to fracture of the green body, by end capping or lamination. 


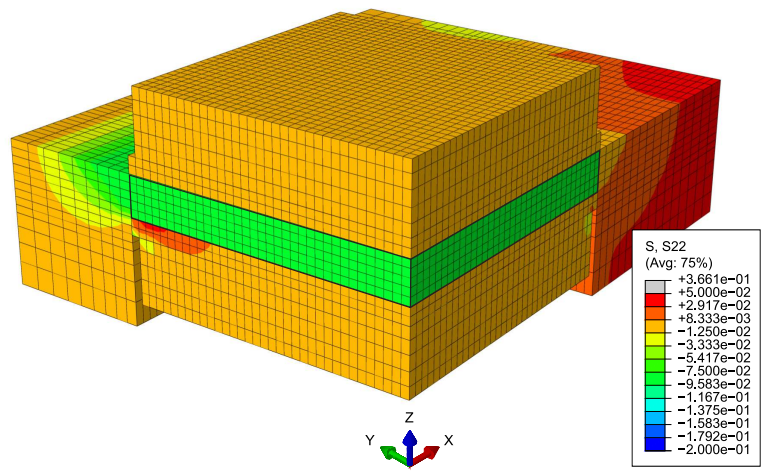

(a) Geostatic step: $\sigma_{y y}$

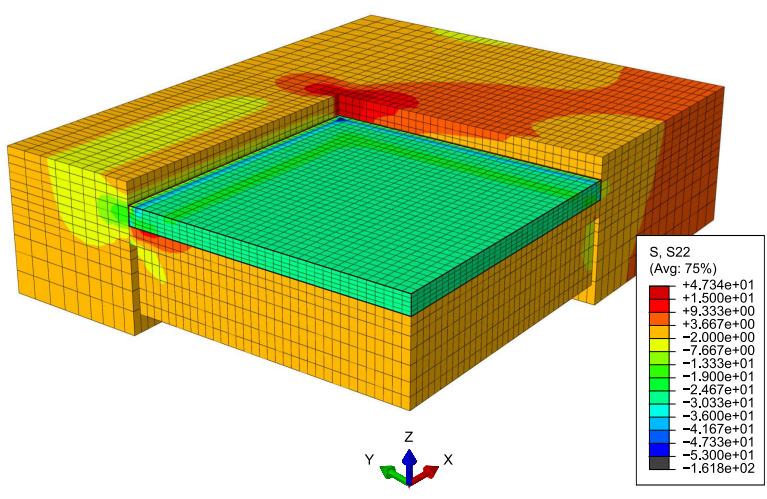

(c) End of compaction phase: $\sigma_{y y}$

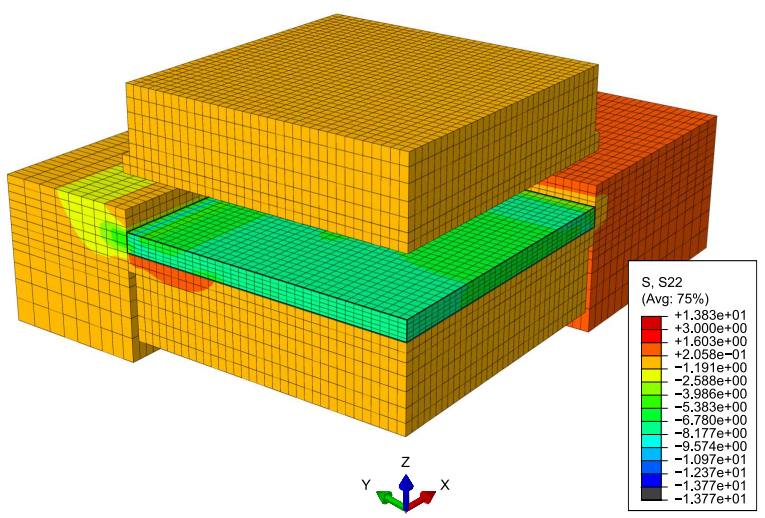

(e) Unloading: $\sigma_{y y}$

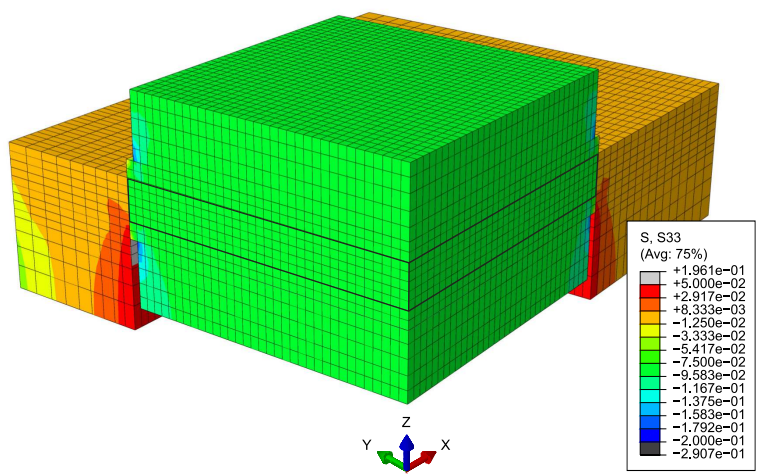

(b) Geostatic step: $\sigma_{z z}$

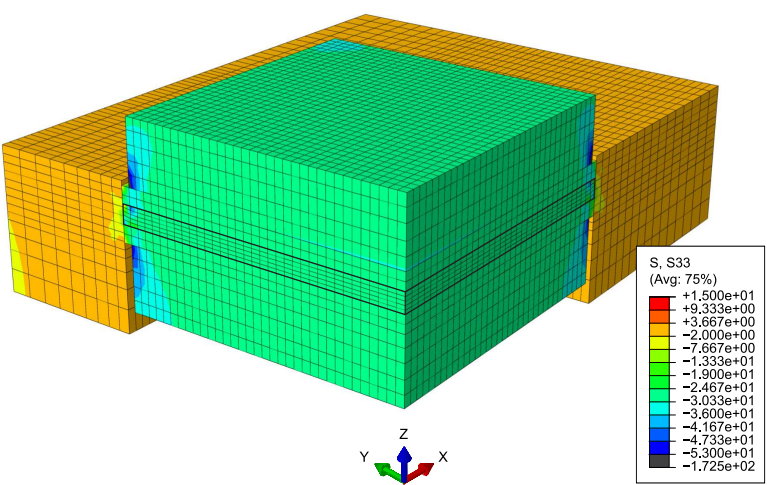

(d) End of compaction phase: $\sigma_{z z}$

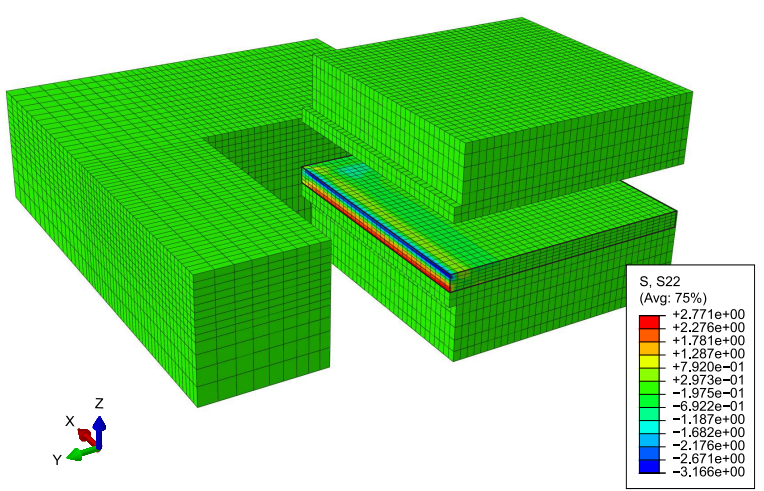

(f) Extraction of the green body: $\sigma_{y y}$

Figure 9: Three-dimensional numerical simulation of industrial tile forming. Stress distribution (lateral stress $\sigma_{y y}$ and axial stress $\sigma_{z z}$ ) in the ceramic powder and in the mould (composed by matrix, upper and lower plates) at the end of the main stages of powder compaction: geostatic step (upper part), axial loading (central part), axial unloading (lower part, left) and extraction of the tile (lower part, right). 


\subsection{Estimation of transversal load on the lateral die wall}

All FE analyses denoted high contact pressure values on the steel matrix. For an applied axial load of $45 \mathrm{MPa}$, three-dimensional and two-dimensional simulations yielded respectively average transversal pressure values equal to $37.73 \mathrm{MPa}$ and to $39.45 \mathrm{MPa}$, respectively. The contact pressure for the three-dimensional simulation is shown in Figure 11 (upper part). The average contact pressure has been calculated dividing the resultant of the nodal contact forces by the contact area.

These values of lateral contact pressure on the die wall are much higher than those usually considered in the design of tile forming devices. In the industrial practice, the lateral contact pressure is empirically assumed to be one sixth of the axial pressure applied on the powder.

To clarify this point, a specific experimental investigation has been performed, by placing a pressure-sensitive Fuji Prescale MS film between ceramic powder and steel matrix, see Fig. 10.
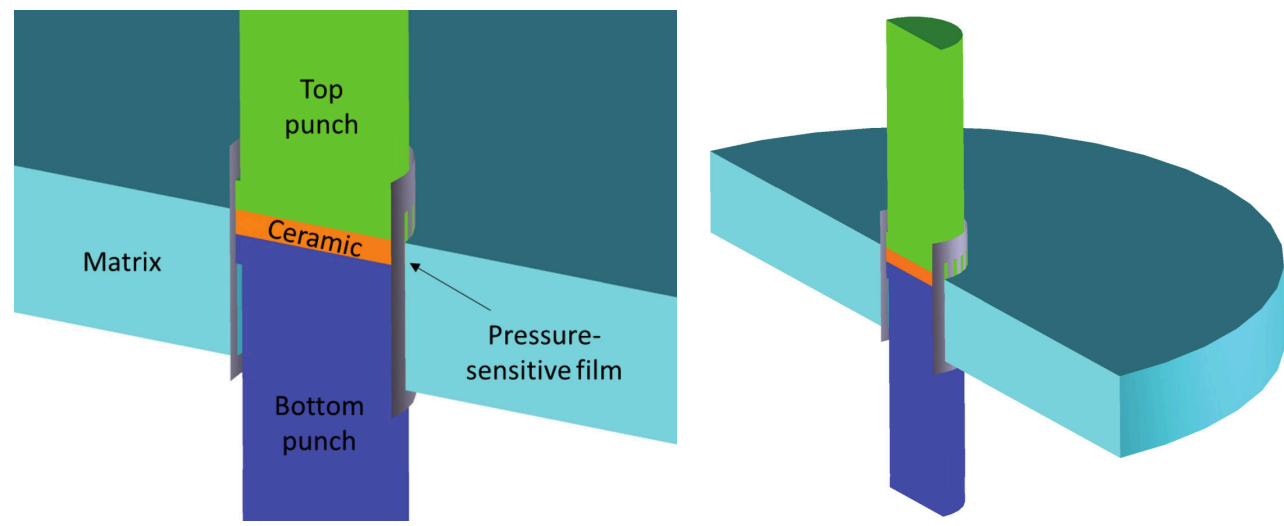

Figure 10: Description of the performed experimental test: the pressure-sensitive film is placed between the ceramic powder and the steel matrix.

This film is composed of a polyester base on which a colour-developing material is coated, with the micro-encapsulated colour-forming material layered on top. When pressure is applied on the film, the microcapsules are broken and the colour-forming material reacts with the colour-developing material, and this process causes magenta colour forming. Microcapsules are designed to react to various degrees of pressures, releasing their colour forming material at a density that correspond to specific levels of applied pressure.

Figure 11 (lower part) shows the imprint left by the ceramic powder on the pressure-sensitive film. The lateral pressure on the matrix was obtained by analysing the image using a dedicated program that convert magentascale to RGB values. For a forming axial pressure of $45 \mathrm{MPa}$, the measured average lateral pressure on the matrix is equal to $39 \mathrm{MPa}$, confirming the results of the simulations with a very good accuracy. 

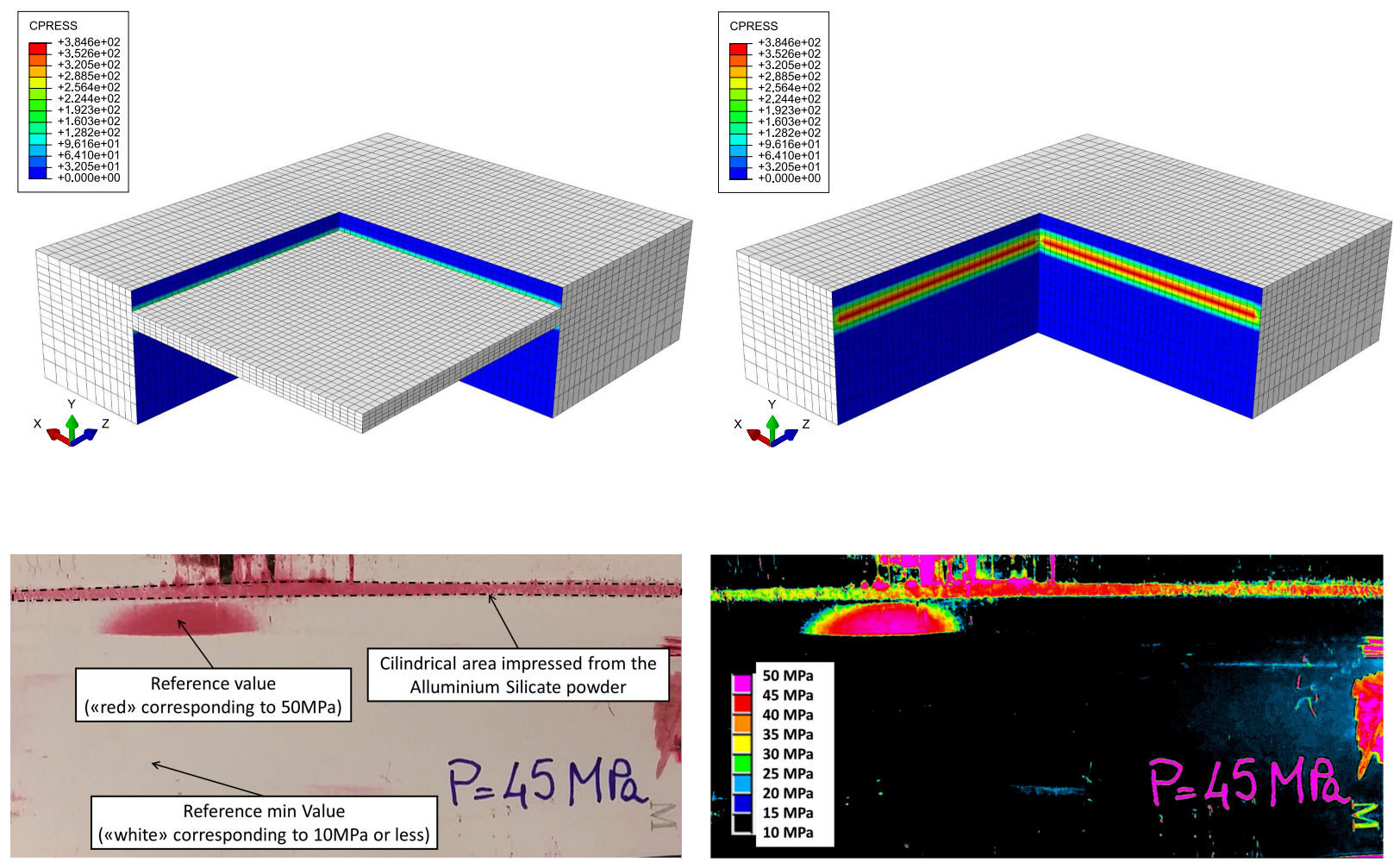

Figure 11: Contact pressure values in the three-dimensional simulation (upper part) and imprint left on the pressure-sensitive film after the experimental test (lower part). 


\subsection{Density distribution in a combed finish tile after die pressing}

The density distribution in green bodies is of primary importance in the optimization of tile forming processes. In fact, non-uniform density can affect the subsequent sintering process, resulting in a poor quality of the final ceramic product. Figure 12 shows the void ratio distribution in a combed finish tile green body, predicted by the finite element simulation. The void ratio, defined as the ratio of the volume of voids to the volume of solid, $e=V_{V} / V_{S}$, is related to the relative density by

$$
\frac{\rho}{\rho_{0}}=\frac{1+e_{0}}{1+e}
$$

where $\rho_{0}$ and $e_{0}$ are the initial values. We notice higher void ratio values, corresponding to lower density, in correspondence of the protrusions. These results pave the way to more advanced virtual prototyping analyses, aiming at the optimization of the design of tile forming devices, in order to produce green bodies with improved density distribution.

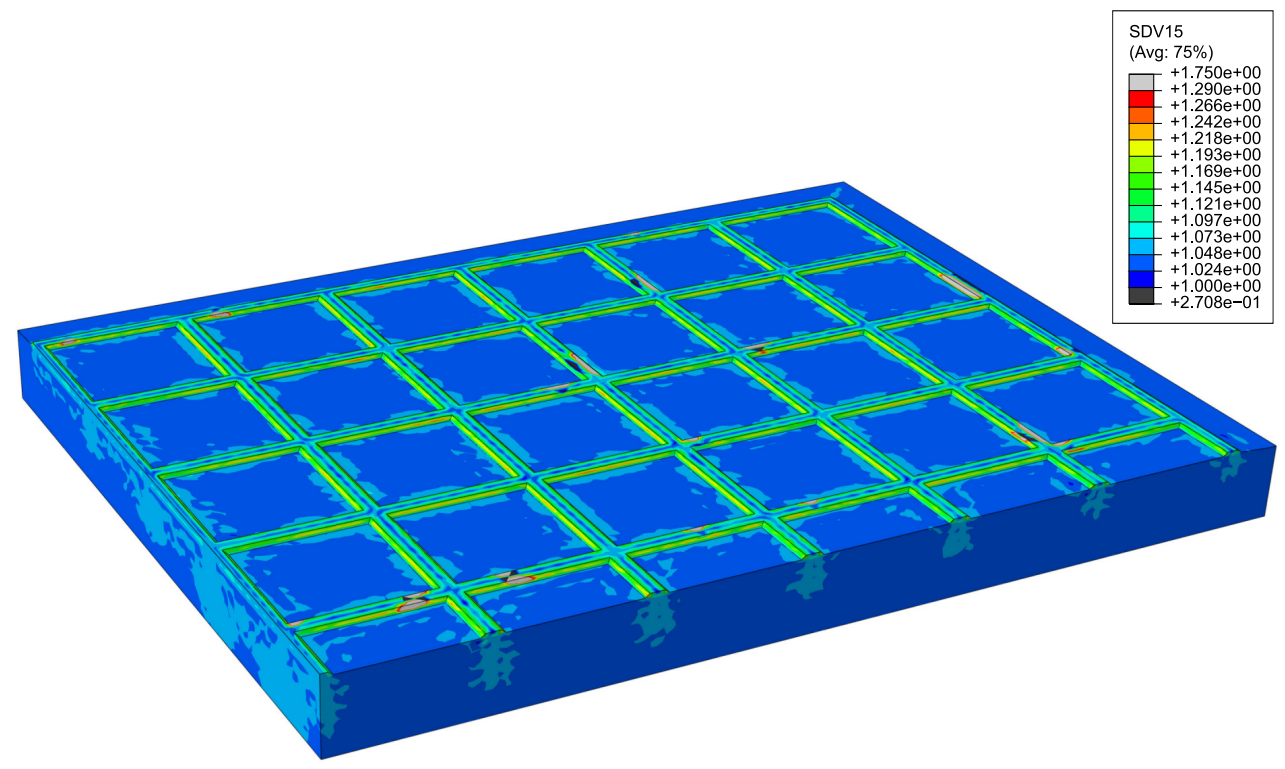

Figure 12: Void ratio distribution in a combed finish tile after die pressing.

\section{Conclusions}

A simulation tool has been presented for the design and the optimization of a forming process of ceramic powders for the production of tiles. In the modelling composition, granulometry, morphology, and humidity of the powder is accounted for in a phenomenological perspective, namely, through the introduction of constitutive parameters obtained from specifically-designed experimental tests. Other parameters needed for the analyses were shown to be deductible through a multi target optimization software. All these parameters allow for a refined description of the complex mechanical behaviour of the material occurring during cold forming. The software developed for the in-silico 
evaluation of the forming processes has been proven to be numerically robust and has been validated through a comparison between numerical predictions and experimental measurements of the pressure to which a tile is exposed during forming. The availability of the simulation tool opens new possibilities in the design of manufacturing ceramic pieces and in the optimization of the production process to achieve higher production quality requirements in terms of density distribution, residual stresses, dimension tolerance, cracking, and minimization of effects related to the spring-back.

\section{Acknowledgements}

LA, MC, and DM acknowledge financial support from the European Union's Seventh Framework IAPP Programme PIAP-GA-2011-286110-INTERCER2. MP and FDC acknowledge financial support from the European Union's Seventh Framework Programme PIAPP-GA-2013-609758HOTBRICKS. AP acknowledges financial support from the European Union's Seventh Framework Programme PITN-GA-2013-606878-CERMAT2.

\section{References}

[1] P. Cundall and O. Strack, "Discrete numerical model for granular assemblies", Geotech. 29 (1979), no. 1, 47-65.

[2] P. Cundall, "Formulation of a three-dimensional distinct element model-part i. a scheme to detect and represent contacts in a system composed of many polyhedral blocks", Int. J. Rock Mech. Min. Sci. 25 (1988), no. 3, 107-116.

[3] R. Hart, P. Cundall, and J. Lemos, "Formulation of a three-dimensional distinct element model-part ii. mechanical calculations for motion and interaction of a system composed of many polyhedral blocks", Int. J. Rock Mech. Min. Sci. 25 (1988), no. 3, 117-125.

[4] S. Nosewicz, J. Rojek, K. Pietrzak, and M. Chmielewski, "Viscoelastic discrete element model of powder sintering", Powder Technol. 246 (2013) 157-168.

[5] J. Rojek, S. Nosewicz, K. Pietrzak, and M. Chmielewski, "Simulation of powder sintering using a discrete element model", Acta Mech. et Autom. 7 (2013), no. 3, 175-179.

[6] C. Thornton and J. Lanier, "Uniaxial compression of granular media: numerical simulations and physical experiment", in "Powders \& Grains 97", Behringer and e. Jenkins, eds., pp. 223-226. A.A.Balkema/Rotterdam/Brookfield, 1997.

[7] J.-F. Jerier, B. Hathong, V. Richefeu, B. Chareyre, D. Imbault, F.-V. Donze, and P. Doremus, "Study of cold powder compaction by using the discrete element method", Powder Technol. 208 (2011), no. 2, 537-541.

[8] C. O'Sullivan, J. Bray, and L. Cui, "Experimental validation of particle-based discrete element methods", in "GeoCongress 2006: Geotechnical Engineering in the Information Technology Age", vol. 2006, p. 5. 2006. 
[9] S. C. Thakur, J. Y. Ooi, and H. Ahmadian, "Scaling of discrete element model parameters for cohesionless and cohesive solid", Powder Technol., 2015 -.

[10] F. Bosi, A. Piccolroaz, M. Gei, F. Dal Corso, A. Cocquio, and D. Bigoni, "Experimental investigation of the elastoplastic response of aluminum silicate spray dried powder during cold compaction", J. Eur. Ceram. Soc. 34 (2014) 2633-2642.

[11] C. Della Volpe, G. Fronza, A. Pedrotti, and S. Siboni, "Una nuova procedura per la determinazione non distruttiva della densità e della porosità di materiali di interesse archeologico", in "Atti del IV Congresso Nazionale AIA", pp. 253-254. 2006.

[12] A. Piccolroaz, D. Bigoni, and A. Gajo, "An elastoplastic framework for granular materials becoming cohesive through mechanical densification. part I - small strain formulation", Eur. J. Mech. A/Solids 25 (2006), no. 2, 334-357.

[13] A. Piccolroaz, D. Bigoni, and A. Gajo, "An elastoplastic framework for granular materials becoming cohesive through mechanical densification. part II - the formulation of elastoplastic coupling at large strain", Eur. J. Mech. A/Solids 25 (2006), no. 2, 358-369.

[14] S. Stupkiewicz, A. Piccolroaz, and D. Bigoni, "Elastoplastic coupling to model cold ceramic powder compaction", J. Eur. Ceram. Soc. 34 (2014), no. 11, 2839-2848.

[15] M. Penasa, A. Piccolroaz, L. Argani, and D. Bigoni, "Integration algorithms of elastoplasticity for ceramic powder compaction.", J. Eur. Cer. Soc. 34 (2014), no. 11, 2775-2788.

[16] S. Stupkiewicz, A. Piccolroaz, and D. Bigoni, "Finite-strain formulation and fe implementation of a constitutive model for powder compaction", Comput. Methods Appl. Mech. Eng. 283 (2015) 856-880.

[17] D. Bigoni and A. Piccolroaz, "Yield criteria for quasibrittle and frictional materials", Int. J. Solids Struct. 41 (2004), no. 11-12, 2855-2878.

[18] A. R. Cooper and L. E. Eaton, "Compaction behavior of several ceramic powders", J. Am. Ceram. Soc. 45 (1962), no. 3, 97-101.

[19] L. P. Argani, D. Misseroni, A. Piccolroaz, Z. Vinco, D. Capuani, and D. Bigoni, "Plastically-driven variation of elastic stiffness in green bodies during powder compaction: Part I Experiments and elastoplastic coupling", ArXiv e-prints, 2015 arXiv:1511.08914.

[20] L. P. Argani, D. Misseroni, A. Piccolroaz, D. Capuani, and D. Bigoni, "Plastically-driven variation of elastic stiffness in green bodies during powder compaction. Part II: Micromechanical modelling", ArXiv e-prints, 2015 arXiv:1511.08934.

[21] T. Hueckel, "On plastic flow of granular and rocklike materials with variable elasticity moduli.", Bull Acad Pol Sci Ser Sci Tech 23 (1975), no. 8, 665-674.

[22] T. Hueckel, "Coupling of elastic and plastic deformations of bulk solids", Meccanica 11 (1976), no. 4, 227-235. 
[23] J. Dougill, "On stable progressively fracturing solids", Z. Angew. Math. Phys. 27 (1976), no. 4, 423-437.

[24] D. Bigoni, "Nonlinear solid mechanics. bifurcation theory and material instability", Cambridge University Press, Cambridge, 2012.

[25] S. Stupkiewicz, R. Denzer, A. Piccolroaz, and D. Bigoni, "Implicit yield function formulation for granular and rock-like materials", Comput. Mech. 54 (2014), no. 5, 1163-1173.

[26] F. Poltronieri, A. Piccolroaz, D. Bigoni, and S. Romero Baivier, "A simple and robust elastoplastic constitutive model for concrete", Eng. Struct. 60 (2014) 81-84.

[27] R. Brannon and S. Leelavanichkul, "A multi-stage return algorithm for solving the classical damage component of constitutive models for rocks, ceramics, and other rock-like media", Int. J. Fract. 163 (2010), no. 1-2, 133-149.

[28] A. Pérez-Foguet, A. Rodríguez-Ferran, and A. Huerta, "Consistent tangent matrices for substepping schemes", Comput. Methods Appl. Mech. Eng. 190 (2001), no. 35-36, 4627-4647.

[29] X. Tu, J. Andrade, and Q. Chen, "Return mapping for nonsmooth and multiscale elastoplasticity", Comput. Methods Appl. Mech. Eng. 198 (2009), no. 30-32, 2286-2296.

[30] J. Korelc, "Multi-language and multi-environment generation of nonlinear finite element codes", Eng. with Comput. 18 (2002), no. 4, 312-327.

[31] J. Korelc, "Automation of primal and sensitivity analysis of transient coupled problems", Comput. Mech. 44 (2009), no. 5, 631-649.

[32] B. Adams, L. Bauman, W. Bohnhoff, K. Dalbey, M. Ebeida, J. Eddy, M. Eldred, P. Hough, K. Hu, J. Jakeman, L. Swiler, and D. Vigil, "Dakota, a multilevel parallel object-oriented framework for design optimization, parameter estimation, uncertainty quantification, and sensitivity analysis: Version 6.2 user's manual", Sandia Tech. Rep., 2015. 